\title{
MULTIDISCIPLINARY APPROACH TO CHRONIC CONSTIPATION ASSOCIATED WITH FECAL INCONTINENCE IN CHILDREN
}

\author{
Claudia Olaru ${ }^{1}$, Smaranda Diaconescu ${ }^{1}$, Valeriu V. Lupu ${ }^{1}$, Nicoleta Gimiga ${ }^{1}$, \\ Gabriela Paduraru ${ }^{1}$, Ancuta Ignat ${ }^{1}$, Radian A. Olaru ${ }^{2}$, Vasile Drug ${ }^{3}$, \\ Marin Burlea ${ }^{1}$ \\ ${ }^{1}$ Department of Pediatrics, ,,Sf. Maria“ Children Emergency Hospital, \\ „, Gr. T. Popa “ University of Medicine and Pharmacy, Iasi \\ ${ }^{2}$, , Gr. T. Popa “ University of Medicine and Pharmacy, Iasi \\ ${ }^{3}$ Institute of Gastroenterology and Hepatology, \\ „, Gr. T. Popa“ University of Medicine and Pharmacy, Iasi
}

\begin{abstract}
Constipation is a common symptom in clinical practice. Definition include abnormal transit difficulty during defecation and abnormal stool consistency. Limited diagnostic methods and the many diseases that lead to symptoms, leading to more cases of drug resistance. Treatment includes dietary and behavioral approaches, pharmacological therapy and surgery in carefully selected cases. Surgery is recommended in patients with severe constipation resistant to conservative treatment. Confirmation of indication for surgical treatment requires coIonic transit studies, defecation function and conducting anorectal manometry. Therefore, a close collaboration between pediatric gastroenterologists and surgeon pediatricians is essential for the careful preoperative evaluation and gastrointestinal function. Postoperative complications include intestinal obstruction, abdominal pain, flatulence and diarrhea. The effectiveness of surgery and prevalence of postoperative complications are determined by a careful preoperative evaluation of the gastrointestinal function.
\end{abstract}

Keywords: organic constipation, encomprezis, surgical treatment, child

Chronic constipation is a debilitating disease that is best managed through a multidisciplinary approach that includes pediatricians and gastroenterologists and usually consists of diet modification, cathartic agents, enemas and psychotherapy. However, $40-50 \%$ of patients do not respond to medical treatments, so the surgery orientation has played an increasingly important role over the last decade $(1,2)$. In the past, the surgical approach was limited to the treatment of complications of constipation and correction of anorectal anomalies. Recently, this role has expanded and surgical treatments now include: internal sphincter myomectomy and access for antegrade enemas by right cecostomy. For diagnostic accuracy, transanal rectal biopsy is practiced. (3-6)
According to Rome III criteria (2006), chronic constipation is defined by: the presence of 2 or fewer bowel movements per week; at least 1 episode of fecal incontinence per week; a history of adoption of voluntary retention posts or excessive retention of feces; difficult removal or disposal of faeces of increased consistency; presence of faeces in the rectum; removal of faeces of increased diameter. The diagnosis is supported in children aged 0-4 years if at least two criteria were present in at least 1 month and children over 4 years if at least two criteria were present at least 1 time per week for a minimum of 2 months. (1)

Intestinal fecal incontinence is the loss of control, resulting in involuntary passage of stool. This 
can range from an occasional leakage of small amounts of stool and passing gas up to a complete loss of control of stool. Faecal incontinence is estimated to affect between $0.8-4.1 \%$ of children in Western societies. $(7,8)$ Recent studies in Asia have shown that faecal incontinence is a significant problem in Iran, South Korea and Sri Lanka, ranging from $2 \%$ to $7.8 \%$. (9-11) A recent study from Sri Lanka conducted on children aged 10-16 years reported a higher prevalence of fecal incontinence in younger children $(5.4 \%)$, while in adolescents a much lower prevalence $(<1 \%)$ was reported. (11) A study from the Netherlands also noted that children aged 5-6 years are more likely to develop faecal incontinence than children aged 11-12 years (7). Chronic constipation with faecal incontinence in order of the frequency of cases is associated to the following categories of malformation: anorectal malformation, Hirschsprung's disease, spina bifida or agenesis of the sacrum. Until recently, faecal incontinence and constipation were considered psychiatric disorders and some of these children were directed to psychiatric clinics. In fact, $5.7 \%$ of children attending a psychiatric unit suffer from faecal incontinence. (12) In previous studies the prevalence of fecal incontinence was significantly higher in boys, with a ratio ranging from 3:1 to 6: 1 against girls. (7,11,13-17)

Intestinal motility is one of the most complex and sophisticated functions in the human body. The colon absorbs water and acts as a reservoir. The liquid waste produced by the small intestine in the cecum, turns into solid stool in the descending and sigmoid colon. To achieve fecal continence, three components are needed: rectal sensation, colonic motility and anal sphincter control. The patient's ability to maintain fecal continence vary depending on the type of anorectal malformation with which he/she was born. Major malformations are often accompanied by muscle atrophy, while small malformations are usually associated with good muscle function.

Anorectal defects associated with good prognosis are: ano-vestibular fistula, perineal fistula, rectal atresia, recto-urethral fistula and anal non-perforation without fistula.

Supervision of these children is needed to avoid constipation, impaction and fecal incontinence.

Anorectal defects associated with a poor prognosis are: large cloaca with a common channel largern than three centimeters and recto-vesical fistula.

In children with anorectal malformations some or all of these essential components are missing.
Faecal incontinence is a devastating problem affecting approximately $25 \%$ of patients born with anorectal malformations (1), some patients who underwent surgery for Hirschsprung disease (2), patients who were born with pelvic tumors $(3,4)$, spina bifida (5), sacral agenesis $(6,18)$ and in patients who had severe pelvic trauma. Children and adults with faecal incontinence are often discriminated against and can not have an active and productive life. (19)

Children who underwent surgery for Hirschsprung disease have lost some of these components due to surgery. They often develop postoperative constipation. Of these, a low percentage may suffer from faecal incontinence as a sequela of surgery. Children with constipation after correction of anal imperforation are included in this category.

Children with recto-prostatic fistula may develop in a percentage similar to voluntary bowel control or they may be incontinent. In these cases it is necessary to attempt to make use of the toilet training. If this proves to be unsuccessful, the management of bowel control should be implemented immediately, so that the child can stay clean and avoid the psychological sequelae

Other complications are represented by urinary incontinence that occurs in male children with anorectal defects when they have a highly defective or absent sacrum or where the basic principles of surgery are not observed, significantly damaging the nerve endings during surgery.

Two surgical procedures have been described: colon resection and access to perform retrograde enemas.

According Lahr et al. (18), surgical treatment of constipation can be taken into account if several criteria are met, including: 1) it must justify the severity of surgical risk, 2) medical and psychological causes were excluded, 3) medical treatment failed, and 4) diagnostic studies show anatomical or physiological abnormalities which can be corrected.

Surgical treatment is indicated in selected patients with chronic constipation resistant to pediatric treatment. Patients with constipation as a symptom of an organic disease of the digestive tract where surgery is a treatment of choice must be excluded: slow transit constipation, rectocele, enterocel, rectal intussusception and rectal prolapse.

Preoperative evaluation should include: intestinal transit time, anorectal manometry, antroduodenal manometry, anal sphincter electromyography and defecography. 
Bowel management is indicated in patients with faecal incontinence after surgical repair of imperforate anus or Hirschsprung disease. It is also used in patients who present continence after surgery, but suffer from constipation (18).

Certain categories of patients may be candidates for resection of sigmoid. This procedure is indicated for those patients with a massive expansion of the rectosigmoid (Fig. 1). The goal of resection is to reduce the amount of the laxatives that are needed to empty the colon on a daily basis, improving thus the quality of life. In this group of patients mesosigmoid is revealed by the use of contrast enemas. The intervention involves partial resection of the dilated part of colon, while the non-dilated part is assumed of normal motility, and is used to perform anastomosis to the rectum. Distal rectum is preserved.

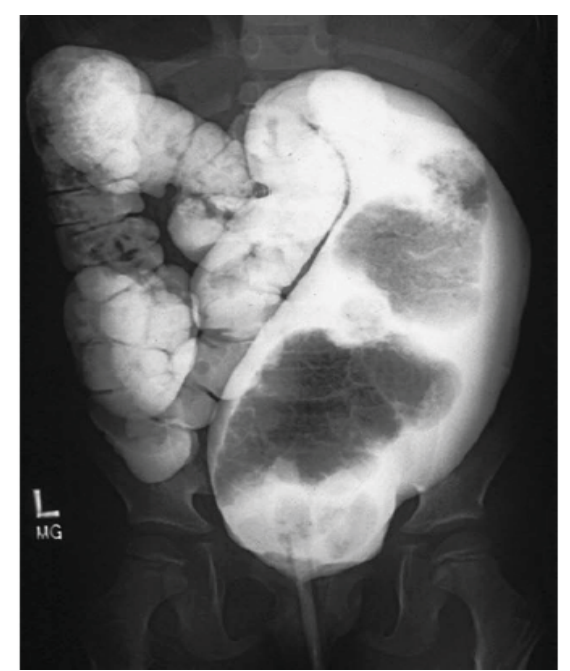

FIGURE 1. Irigography: fecal incontinence in a patient. Colonic dilatation. (25)

Patients to which resection therapy is recommended must show fecal continence. Bowel resection in a patient with incontinence can worsen the condition, moving from the tendency to form solid stool to have free stool. When sigmoid is removed, the patient becomes incontinent with loose stools, a condition that it is more difficult to manage. $(20,21)$

Therefore, sigmoid resection is used only in patients who can voluntarily empty the colon, albeit with massive doses of laxatives. These patients require much lower doses of laxatives after surgery.

It was found that the prognosis was better in patients who had a localized form of megarectosigmoid. Patients with generalized forms did not respond as well to resective therapy. They may require the resection of a larger segment of colon, sigmoid colon resection and anastomosis of the transverse colon. (20)
Sigmoid resection complications include leakage at the anastomosis level. This complication is similar to the complication that may arise after colon resections for other conditions.

In patients with faecal incontinence, fair treatment consists of a bowel management program by administering enemas on a daily basis. Their rectal administration can be problematic in older children. They tend to gain a degree of independence and they do not want that the administering of enemas to be done by parents. In these patients, appendicostomy (Malone procedure) or cecostomy for anterograde administration of enemas may be performed. (19) (Fig. 2)

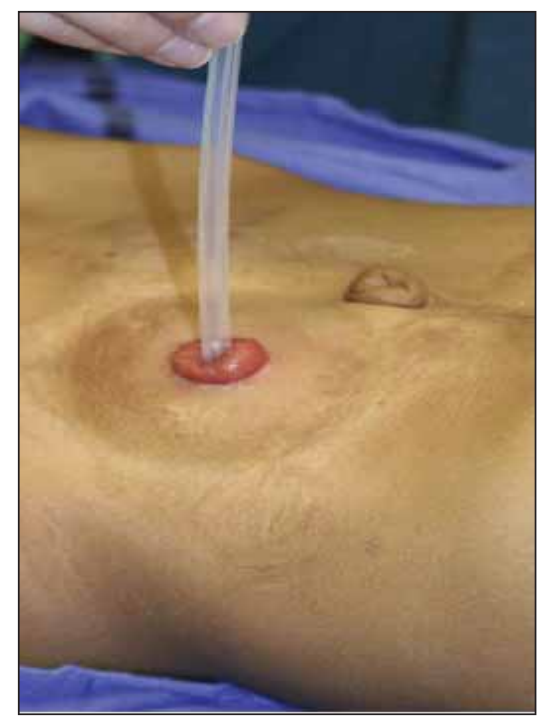

FIGURE 2. Anterograde enema. (25)

The intervention involves connecting to the abdominal wall and molding a valve mechanism that allows catheterization, but avoids leakage of stool.

A study of 32 patients who were treated with antegrade enemas showed fewer episodes of faecal incontinence and abdominal pain and improved mood. The disadvantages of this process are that some patients have complications related to the stoma, such as stenosis, leakage, mucus, fecal leakage and pain. (22) Improved surgical techniques such as laparoscopic placement of the tube make the procedure easier and more acceptable to patients.

Follow-up and regular reassessment is required. Often, the enema volume must be adjusted. Rectal examination and abdominal radiography helps to assess the efficacy of the procedure.

The prognosis for children with faecal incontinence is generally variable. One study showed that approximately $50 \%$ of affected children developed at least one relapse during the first 5 years after initial remission. (23) In another study, the authors 
found that at age 16, one-third of children were still symptomatic, indicating the possibility of progression to adulthood. (23) However, a recent systematic review found that most children with faecal incontinence recovered within 6-12 months after initiation of treatment and recovery did not depend on age of onset, family history and disease severity. (24)

\section{CONCLUSIONS}

In selected patients, surgical intervention in cases of severe constipation can be a valuable method of treatment. In case of failure of medical or in case of severe malformations, surgical treatment is rec-

\section{REFERENCES}

1. Ruttenstock E., Puri P. A meta-analysis of clinicăl outcome in patients with total intestinal aganglionosis. Pediatr Surg Int. Oct 2009; 25(10):833-9.

2. Theocharatos S., Kenny S.E. Hirschsprung's disease: current management and prospects for transplantation of enteric nervous system progenitor cells. Early Hum Dev. Dec 2008; 84(12):801-4.

3. Bekkali N.L., van den Berg M.M., Dijkgraaf M.G., et al. Rectal fecal impaction treatment in childhood constipation: enemas versus high doses oral PEG. Pediatrics. Dec 2009; 124(6):e1108-15.

4. Bongers M.E., van den Berg M.M., Reitsma J.B., Voskuijl W.P., Benninga M.A. A randomized controlled trial of enemas în combination with oral laxative therapy for children with chronic constipation. Clin Gastroenterol Hepatol. Oct 2009; 7(10):1069-74.

5. Marques G.M., Martins J.L., Nobre V.D. Comparison between perfusion and balloon techniques for performing anorectal manometry in children with intestinal constipation. Acta Cir Bras. Sep-Oct 2008; 23(5):405-11.

6. Levitt M.A., Pena A. Pediatric fecal incontinence: a surgeon's perspective. Pediatr Rev. Mar 2010; 31(3):91-101.

7. Joinson C., Heron J., Butler U., von Gontard A. Psychological differences between children with and without soiling problems. Pediatrics 2006; 117: 1575-84.

8. van der Wal M.F., Benninga M.A., Hirasing R.A. The prevalence of encopresis în multicultural population. J Pediatr Gastroenterol Nutr 2005; 40: 345-8.

9. Sohrabi S., Nouraie M., Khademi H., Bagqhizadeh S., NasseriMoghaddam S., Malekzadeh R. Epidemiology of uninvestigated gastrointestinal symptoms in adolescents: A populationbased study applying the Rome II questionnaire. J Pediatr Gastroenterol Nutr 2010; 51: 41-5.

10. Chung J.M., Lee S.D., Kang D.I., et al. An epidemiologic study of voiding and bowel habits in Korean children: a nationwide multicenter study. Urology 2010; 76: 215-9.

11. Rajindrajith S., Devanarayana N.M., Benninga M.A. Constipationassociated and nonretentive fecal incontinence in children and adolescents: an epidemiological survey in Sri Lanka. J Pediatr Gastroenterol Nutr 2010; 51: 472-6. ommended. The effectiveness of surgery and the prevalence of postoperative complications are determined by a very careful preoperative evaluation of the gastrointestinal function. Before surgery all available methods of conservative treatment recommended should be used. Favorable prognosis will be influenced by the absence of postoperative complications or the care given by the postoperative team: surgeon, pediatrician and gastroenterologist.

Acknowledgements: We thank the staff of the Department of Gastroenterology Pediatric Hospital "Sf. Maria ", Iasi
12. Olatawura M.O. Encopresis, a review of thirty-two cases. Acta Pediatr Scand 1973; 62: 358-64.

13. Levine M.D. Children with encopresis: a descriptive analysis. Pediatrics 1975; 56: 412-6.

14. Voskuijl W.P., Heijmans J., Heimans H.S., Taminiau J.A., Benninga M.A. Use of Rome II criteria în childhood defecation disorders: applicability în clinicăl and research practice. J Pediatr 2004; 145: 213-7.

15. Loening-Baucke V. Encopresis and soiling. Pediatr Clin North Am 1996; 43: 279-98.

16. Berg I., Jones K.V. Functional faecal incontinence în children. Arch Dis Child 1964; 39: 465-72.

17. Voskuijl W.P., Reitsma J.B., van Ginkel R., Buller H.A., Taminiau J.A., Benninga M.A. Longitudinal follow up of children with functional nonretentive fecal incontinence. Clin Gastroenterol Hepatol 2006; 4: $67-72$

18. Levitt M.A., Pena A. Surgery and constipation: when, how, yes, or no. J Pediatr Gastroenterol Nutr. Sep 2005; 41 Suppl 1:S58-60.

19. Levitt M.A., Soffer S.Z., Pena A. Continent appendicostomy in the bowel management of fecally incontinent children. J Pediatr Surg. Nov 1997; 32(11):1630-3.

20. Pena A., Levitt M.A. Colonic inertia disorders in pediatrics. Curr Probl Surg. Jul 2002; 39(7):666-730.

21. Pena A., Guardino K., Tovilla J.M., Levitt M.A., Rodriguez G., Torres R. Bowel management for fecal incontinence in patients with anorectal malformations. J Pediatr Surg. Jan 1998; 33(1):133-7.

22. Marshall J., Hutson J.M., Anticich N., Stanton M.P. Antegrade continence enemas in the treatment of slow-transit constipation. J Pediatr Surg 2001; 36: 1227-30.

23. van Ginkel R., Reitsma J.B., Buller H.A., van Wijk M.P., Taminiau J.A., Benninga M.A. Childhood constipation: Iongitudinal follow-up beyond puberty. Gastroenterology 2003; 125: 357-63.

24. Pijpers M.A., Bongers M.E., Benninga M.A., Berger M.Y. Functional constipation in children: a systematic review on prognosis and predictive factors. J Pediatr Gastroenterol Nutr 2010; 50: 256-68.

25. Peña A., Levitt M.A. Colonic inertia disorders in Pediatrics'. in Curr Probl Surg, Wells SA 